\title{
Registration of 3D Photographs with Spiral CT Images for Soft Tissue Simulation in Maxillofacial Surgery
}

\author{
Pieter De Groeve ${ }^{1}$, Filip Schutyser ${ }^{1}$, Johan Van Cleynenbreugel $^{1}$, and \\ Paul Suetens ${ }^{1}$ \\ Medical Image Computing (Radiology - ESAT/PSI), Faculties of Medicine and \\ Engineering, University Hospital Gasthuisberg, Herestraat 49, B-3000 Leuven, \\ Belgium \\ Pieter.DeGroeve@uz.kuleuven.ac.be
}

\begin{abstract}
Prediction of the facial outcome after maxillofacial surgery is not only of major interest for surgeons but also for patients. A mirror-like image of the expected surgical outcome gives important information for the patient and provides the surgeon with a good communication tool. This paper presents a method for registration of 3D photographs with 3D CT images, to provide the patient with a realistic view of the natural complexion of the simulated postoperative outcome of his/her face. A rigid ICP-based registration algorithm, followed by a non-rigid transformation produces a close match between the two surfaces.
\end{abstract}

Keywords Surface registration, soft tissue modeling, image guided therapy, maxillofacial surgery simulation

\section{Introduction}

Prediction of the facial outcome after maxillofacial surgery is not only of major interest for surgeons but also for patients. A mirror-like image of the expected surgical outcome is the goal for soft tissue simulators in maxillofacial planning environments.

Besides realistic modeling of the facial soft tissues, a natural visualization of the results is very important. Currently, CT-imaging is needed as input for maxillofacial planning environments [1]. Bone structures, skin surfaces, geometric descriptions of the facial soft tissues can be extracted from these data. However, when inspecting skin surfaces from CT, is it hard to recognize the patient. This is due to the lack of color information. In fact, we need a skin surface extracted from $\mathrm{CT}$ together with the natural complexion of the face. This paper presents a method to generate 'colored CT skin surfaces'.

$3 \mathrm{D}$ photography acquires both shape and texture. Combining this information together with CT-data provides 'colored CT skin surfaces'. Laser scanners are well-known 3D photography systems. They generate accurate surfaces. However, these systems are very expensive and the acquisition time is long. Other 
systems generate 3D photographs from some 2D photos with a pattern projected. These systems are less expensive, have short acquisition times, but are less accurate.

This paper focuses on an algorithm for registration of 3D photographs acquired by the latter type of systems, and CT surfaces resulting in 'colored CT skin surfaces'. Special attention has to be paid to the robustness of the system because of the different acquisition conditions: lying in a CT-scanner with a rather serious facial expression, and sitting on chair with a smile for the photograph. These elements turn registration into a nontrivial task.

Section 2 describes the registration algorithm. Results are presented in section 3. Concluding remarks finish the paper.

\section{Methods}

For the acquisition of 3D photographs an "active" 3D system is used. Figure 2 shows a face under structured ligth conditions. Surface information is recovered from the deformation of the pattern on the $2 \mathrm{D}$ picture.

On the other hand, the skin surface of the patient is extracted from CT image data by thresholding. The 3D photograph is then registered with the skin surface from using a 3D surface matching algorithm. Our algorithm is based on the Iterated Closest Point (ICP) algorithm, see [2]. Some fine-tuning was needed to meet the constraints of this application.

A first adaptation is needed when the CT skin-surface does not completely covers the surface of the 3D photograph. Then some points of the 3D photograph won't have a corresponding point on the CT surface. For each these points no corresponding points are found. These sets of correspondences must be rejected,to avoid bad convergence. Therefore a constraint is added : two points can only be two corresponding points, if the distance between them is less or equal than some threshold. This treshold is arbitrary chosen with respect to the accuracy of the acquired 3D photorgaph. An other method, using a generalized Mahalanobis distance, is mentioned in 3 .

To improve accuracy in finding corresponding points, not only Euclidean distance, but also difference in normal orientation and difference in principal curvatures can be taken in account, see 3. In our case, only coordinates and normals are used. We define a new distance between a point $P(x, y, z)$ with normal $\left(n_{x}, n_{y}, n_{z}\right)$ and point $Q\left(x^{\prime}, y^{\prime}, z^{\prime}\right)$ with normal $\left(n_{x}^{\prime}, n_{y}^{\prime}, n_{z}^{\prime}\right)$ as :

$$
\begin{aligned}
D(P, Q)= & {\left[\left(x-x^{\prime}\right)^{2}+\left(y-y^{\prime}\right)^{2}+\left(z-z^{\prime}\right)^{2}\right]+} \\
& \alpha *\left[\left(n_{x}-n_{x}^{\prime}\right)^{2}+\left(n_{y}-n_{y}^{\prime}\right)^{2}+\left(n_{z}-n_{z}^{\prime}\right)^{2}\right]
\end{aligned}
$$

where $\alpha$ is a weight factor, controlling the importance of difference in normal orientation.

A 3D search data-structure is applied to find corresponding points in a fast way. The 3D-space is equally divided in cubes, all of the same dimensions. Every point of the 3D CT-data belongs to one cube. For each point of the 3D photograph corresponding points are searched for only in the cubes surrounding the 
point. An other technique using k-D trees is proposed in [4]. For further speed improvement, the data can be downsampled. To increase accuracy, the data is downsampled only the first few iterations. In this way the initial rough alignment converges rapidly to a better match in the first iterations. Then, without downsampling, the next iterations proceed a more accurately registration between the two surfaces. The rigid registration is stopped if the change between two succeeding transformations is less than some tolerance, or if a maximum number of iterations has been exceeded.

Due to inaccuracies in the 3D photograph, mostly in regions around the eyes, nose and mouth, rigid registration is insufficient, especially when used in surgery simulation, where an almost perfect match with CT-data is required. Therefore a non-rigid transformation is needed for a better and closer match between the two surfaces. Once the best rigid displacement is found, a projection-like transformation is applied by non-rigidly and individually translating every point of the 3D photograph toward the CT surface. The direction for translating a point is found by defining a local normal, given by the average of the normals of the two corresponding points. The distance between the point and the surface is approximated by projecting the Euclidean distance between the point and its corresponding point onto this local normal, see figure 1] After non-rigid transformation the two surfaces match very closely, and texture of the $3 \mathrm{D}$ photograph can be added to the CT skin-surface.

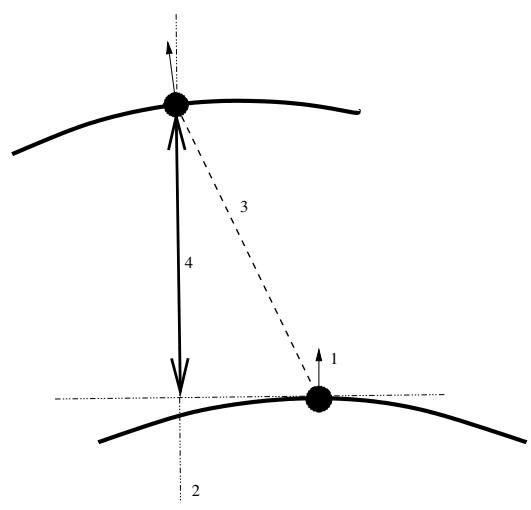

Fig. 1. Non-rigid transformation. From the corresponding points, each with its normal(1), a local normal is calculated(2). The Euclidean distance between the two points(3) is projected on this local normal, resulting in the approximate distance between the point and the surface(4). 


\section{Results}

In this section, we present results of the registration algorithm. In figure 3 the top image represents the two unregistered surfaces. These surfaces must be roughly aligned before the algorithm can start. This is done by interactive manual matching of the two surfaces.

Once initialized, the rigid registration is applied and the images in the second row are obtained. Both surfaces have about 20.000 points. The maximum distance between two corresponding points is chosen to be $5 \mathrm{~mm}$. This should take in account the inaccuracies of the 3D photograph. For speeding up the searching of correspondences, cube dimensions of the search-structure are also $5 \mathrm{~mm}$ in each dimension. This leads to an average of 5 points in each cube. The rigid transformation is found after 50 iterations with downsamplefactor 5 within five seconds CPU time on a SGI Octane R10000 $175 \mathrm{MHz}$, followed by another 50 iterations without downsampling in about 1 minute CPU time. The results are shown on the second row of figure 3 .

We can see, however that the surfaces do not match exactly. For more tightly matching the surfaces, non-rigid transformation is applied (in less then two seconds CPU time), resulting in the images on the third row. This result is used to add the texture of the registrated photograph to the CT-data, shown in the bottom row images. This example illustrates that after rigid registration, the non-rigid displacement is needed for an almost perfect match.

\section{Conclusion}

In this paper we proposed a method for registration of a 3D photograph with CTdata using rigid and non-rigid techniques. Realistic results are obtained which are useful in the domain of maxillofacial surgery planning environments. Only when differences between 3D photograph and CT differ to much, problems might occur in finding correct correspondences, which leads to bad non-rigid transformations. However, efforts to optimize the accuracy of 3D photography and to improve non-rigid registration can alleviate these problems.

\section{Acknowledgments}

The work discussed here belongs, partly to the EU-funded Brite Euram III PISA project (nr. BRPR CT97 0378), a collaboration between Materialise NV, Belgium; Philips Medical Systems BV, the Netherlands: ICS-AD; DePuy International Ltd, UK; Ceka NV, Belgium; K.U. Leuven, Belgium: ESAT/Radiology Div. Biomechanics; University of Leeds, UK: Research School of Medicine, partly to the grant GOA/99/05 (VHS+ Variability in Human Shape and Speech) of the K.U.Leuven Research Council, and to a grant for research specialization from the Flemish Institute for stimulation of the scientific-technological research in the industry (IWT) to Filip Schutyser. 


\section{References}

1. F. Schutyser, J. Van Cleynenbreugel, M. Ferrant, J. Schoenaers, P. Suetens: Imagebased 3D planning of maxillofacial distraction procedures including soft tissue implications. Proceedings 3rd international conference on medical image computing and computer-assisted intervention - MICCAI2000, lecture notes in computer science, vol. 1935, pp. 999-1007, October 11-14, 2000, Pittsburgh, Pennsylvania, USA

2. P.J. Besl, N.D. McKay: A method for registration of 3-D shapes. IEEE Trans. PAMI 14 (2), p. 239-256, 1992.

3. J. Feldmar, N. Ayache: Rigid, affine and locally affine registration of smooth surfaces. INRIA Technical Report No. 2220, 1994.

4. Iterative point matching for registration of free-from curves and surfaces. Int. J. Comp. Vision 13 (2), 119-152.

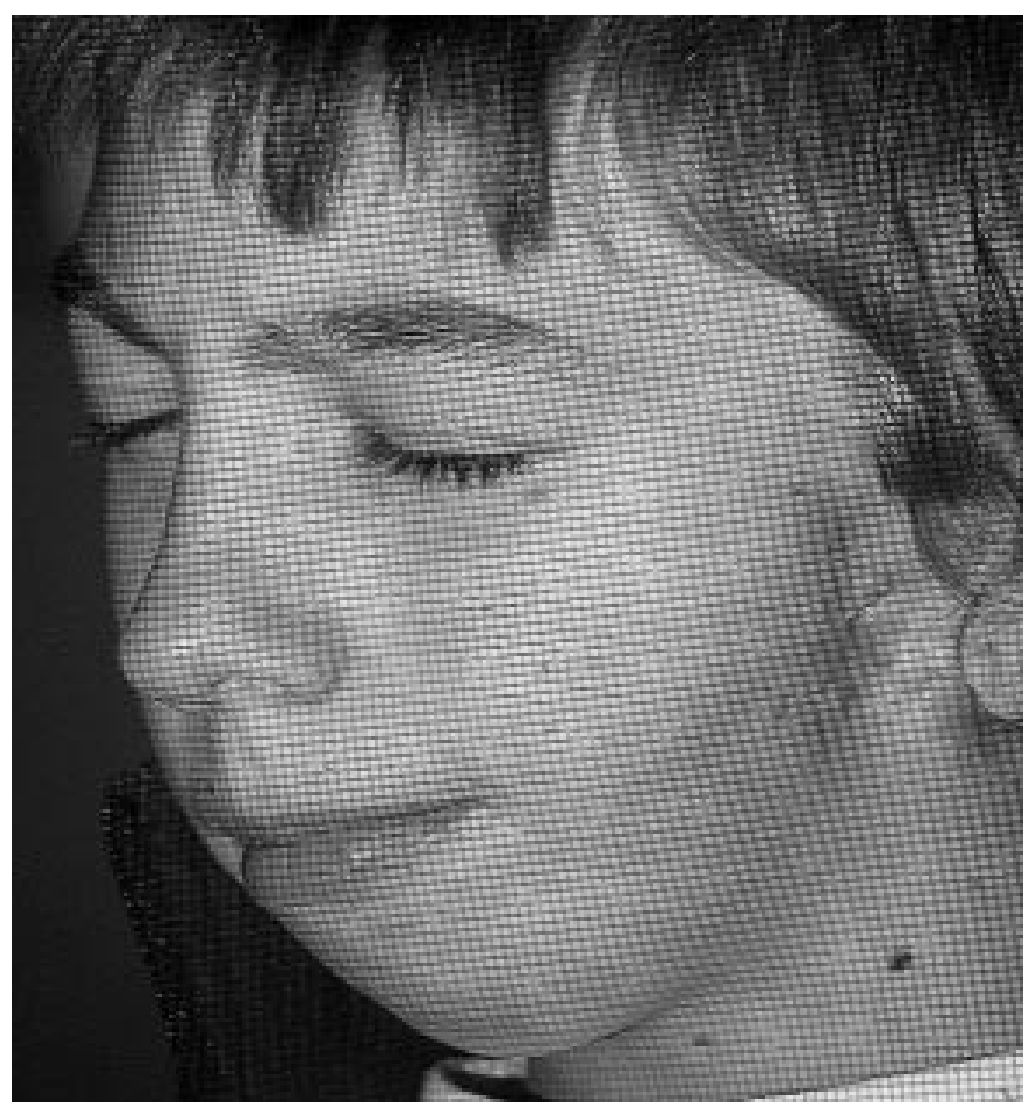

Fig. 2. For the acquisition of 3D photographs structured ligth is used, projecting a pattern onto the face. 3D structure can be extracted from the deformation of this pattern. 

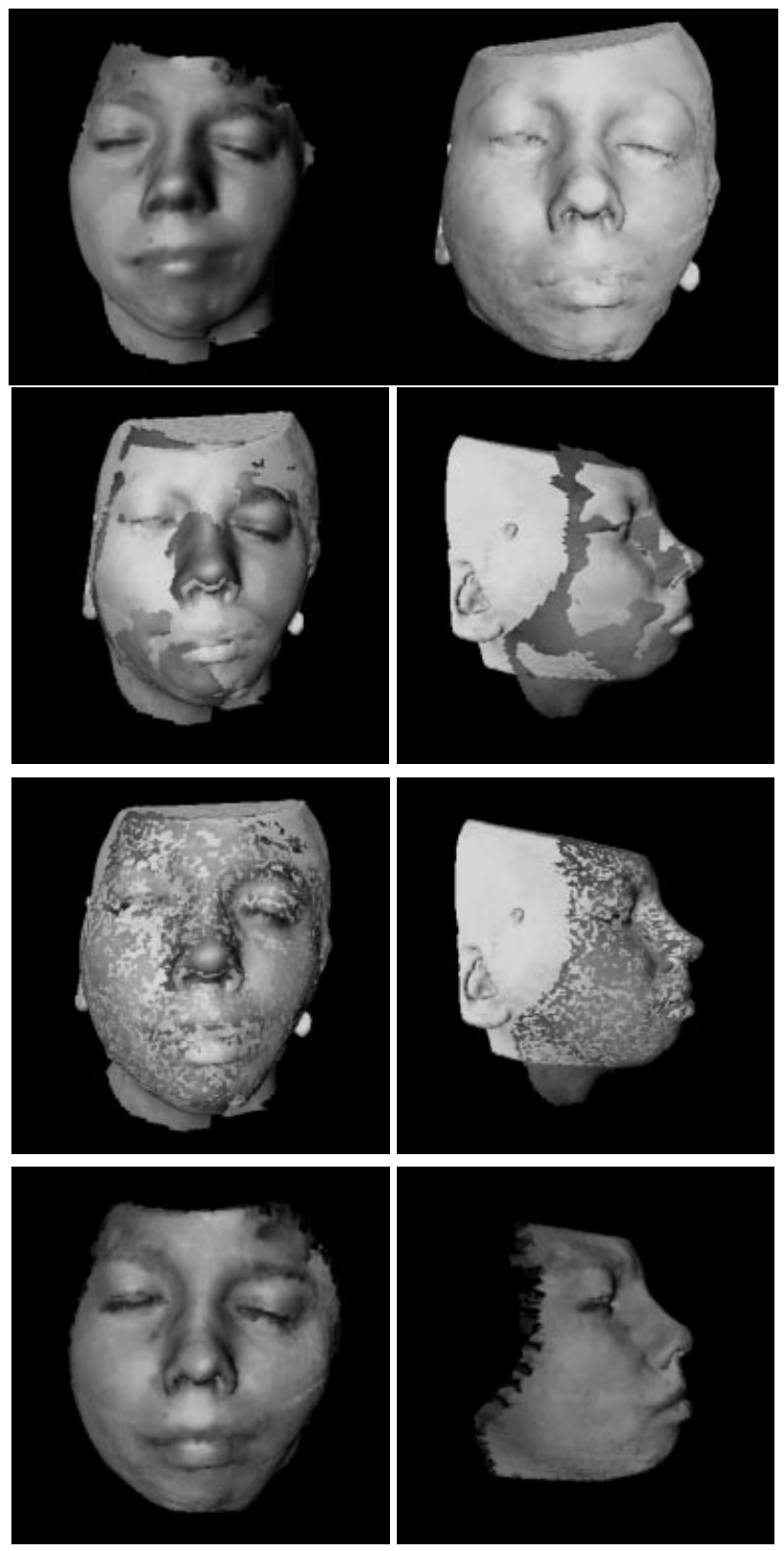

Fig. 3. The skin surfaces from 3D photography and CT imaging are shown on the top image. After rigid registration the middle row images are obtained. Nonrigid registration matches the two surfaces more closely (third row). Based on this result, the texture of the $3 \mathrm{D}$ photograph is added to the CT derived data resulting in the bottom row images. 